\title{
Country Level Preparation for the Deployment of COVID-19 Vaccine
}

\author{
Salah T. Al Awaidy ${ }^{1}$, Chadia Wannous ${ }^{* 2}$, \\ ${ }^{1}$ Office of Health Affairs, Muscat, Ministry of Health, Oman \\ ${ }^{2}$ Future Earth- Health Knowledge Action Network \& Towards A Safer World Network (TASW), \\ Stockholm, Sweden.
}

*Corresponding Author: Chadia Wannous, Future Earth- Health Knowledge Action Network \& Towards A Safer World Network (TASW), Stockholm, Sweden.

\begin{abstract}
COVID-19 vaccine represents one of the key solutions in mitigating the global health crisis instigated by COVID-19 pandemic. In addition to having safe and effective COVID-19 vaccines, it is of utmost importance to, not only accelerate vaccine development and manufacturing but also to ensure equitable distribution and access among and within countries. Containing the global pandemic requires global solidarity and coordination, which is crucial in achieving fair and equitable access to safe and effective vaccine worldwide, and at the same time ensuring that countries are well prepared to implement COVID-19 vaccination programs and efficiently provide COVID-19 vaccine to target groups. The major considerations for the implementation of the COVID-19 vaccine at the country level are: 1. Regulatory and legal issues, 2. Framework for decision making regarding phased vaccination and 3. Programmatic and operational issues. This article briefly highlights these considerations and address the key challenges for COVID-19 vaccine implementation to ensure the vaccines can be safely and timely delivered to all those who need them.
\end{abstract}

Keywords: COVID-19; Deployment; Preparations, Implementation; COVID-19 Vaccine

\section{INTRODUCTION}

Coronavirus disease 2019 (COVID-19), caused by the Severe Acute Respiratory SyndromeCorona Virus 2 (SARS-CoV-2), has significantly impacted every aspect of human life globally. Devastating consequences of this ongoing threat are overwhelming of the health systems worldwide, particularly the fragile health systems in low- and middle-resource settings, freezing of the high interconnected global economy and disrupting essential services and essential immunization which may cause outbreaks of other vaccine-preventable diseases. This disruption has also escalated unemployment in unemployment in many parts of the world(1). As a result of these indirect consequences of the pandemic, every individual in the planet is affected by the pandemic in one way or another.

As of 8 December 2020, WHO reported that there is more than 67 million COVID-19 cases confirmed across the globe with above 1.5 million deaths, and some countries are reporting the second wave of infection (2).
Since the discovery of SARS-CoV-2 virus in December 2019, and availability of its genetic sequence in early January 2020, various research centers, and biotech/pharmaceutical companies have worked relentlessly on different fronts to develop s accurate and rapid diagnostic tests, safe and effective treatment, and vaccine to prevent infection. To date, over two hundred vaccine candidates are in pre-clinical and different phases of clinical development (3). Currently there are 15 vaccine candidates in Phase 3 large-scale trials to test their efficacy. Most recently two mRNA vaccines were effective in helping to prevent COVID-19 with an overall favorable safety profile; Pfizer and its German partner BioNTech released data on November 9 showed that vaccine is $95 \%$ effective. Efficacy was consistent across age, race and ethnicity demographics, and the observed efficacy in adults over 65 years of age was over $94 \%$ (4).

A week later, biotechnology company Moderna announced similar results with effectiveness at 94.5\%(5). On 20 November Pfizer filed for emergency authorization in the US Food and 
Drug Administration (FDA) for its coronavirus vaccine (Pfizer, 2020), and Moderna flowed suit for its COVID-19 vaccine, mRNA-1273. The two vaccines are now approved for full use. The UK has started on 9 December a mass vaccination campaign using doses of the Pfizer-BioNTech vaccine after it received emergency authorization. The first shots will be given to people over age 80 (UK Department of Health and Social Care, 2020). A third promising vaccine The Oxford/AstraZeneca COVID vaccine has efficacy of $90 \%$ in a small group who got a half-dose first, but only $62 \%$ in the majority who received two standard doses, Overall vaccine efficacy across both groups was $70.4 \%$ in a full trial data (6).

Since this is a global pandemic, it is estimated that at least 4.5 billion people will need to be vaccinated in order to reach the optimal herd immunity of $60-70 \%$ (7). Taking into consideration the number of doses needed to vaccinate this large segment of the world population, clearly outlines the necessity to increase manufacturing capacity. If several vaccine candidates prove to be safe and effective, the constraint on a single manufacturing capacity will ease and availability and access will increase. However, this has to be tied to just and equal distribution to achieve effective control of the pandemic. In addition, lifting of nonpharmaceutical interventions, which are crucial in slowing down the spread of the coronavirus, but not able to control it sustainably (8) would be possible allowing social and economic lives to resume and for post pandemic recovery to start.

Key Considerations for Implementingcovid19 Vaccination Programs

WHO Member States have explicitly indicated that implementation of COVID-19 vaccination programs during the pandemic requires rigorous planning and coordination of activities and resources ahead of time to ensure all stakeholders at the country level are aligned and prepared for a smooth roll-out of the vaccination programs, including contingency plans to overcome unforeseen challenges, and to ensure positive reception of the public at large to increase its acceptance and demand (9). Prioritization efforts by Member States is key for phased approach to target priority groups for different levels of vaccine availability and epidemiologic settings. A WHO interim Guidance on developing a national deployment and vaccination plan for COVID-19 vaccines is recently published (10).
In our views, the key consideration in the roll out of COVID19 vaccinations programs at county level can be grouped into three categories: 1) Regulatory and legal issues; 2) Framework for decision-making; and 3) Programmatic and operational issues.

\section{REGULATORY AND LEGAL ISSUES}

\section{Regulatory Review and Approval}

To prepare for COVID-19 vaccine implementation and distribution in a timely fashion, countries are advised to start preparing their regulatory processes in advance of the vaccine market approval for manufacturing, deployment, implementation and monitoring processes. This includes guidance to industry to assist sponsors in the clinical development and licensure of vaccines. For example the US Food and Drug Administration (FDA) guidance includes requirements for : (i) Non-clinical data evaluation; (ii) Clinical protocol evaluation; (iii) Chemistry, manufacturing and quality control; and (iv) a request to receive the regulatory documents from manufacturing countries (11). In addition, the National Regulatory Authorities (NRA) are advised to regularly exchange updates on COVID-19 policies with the international communities to align their review and approval criteria for COVID-19 vaccines with the NRA of other countries, including the US Food and Drug Administration (FDA) and the European Medicines Agency (EMA). This will significantly assist the NRAs in making effective regulatory decisions on the approval of future COVID-19 vaccines.

\section{Legal Agreements}

Previous pandemics have shown that the countries that suffer the most are those with limited or insufficient resources to control the pandemic, including lack of capacity to procure diagnostics equipment, treatments, and vaccines(12).

In the ongoing COVID-19 pandemic, even before any vaccine is approved, many high, upper and lower middle-income countries have already reached bilateral agreements with several vaccine manufacturers for procuring billions of doses of COVID-19 vaccine(13). No evidence suggests that low-income countries have made similar direct deals with vaccine manufactures, indicating that these countries will be entirely reliant on coverage from the COVID-19 vaccine global access (COVAX) facility. 
This situation has ignited the discussion on fair and equitable access to a COVID-19 vaccine. With the leadership and engagement of World Health Organization (WHO), The Vaccine Alliance (Gavi), the Coalition for Epidemic Preparedness Innovations (CEPI), Bill \&Melinda Gates Foundation (BMGF) and donor countries, the Access to COVID-19 Tool (ACT) Accelerator platform was launched in April 2020 with the goal to achieve fair, rapid and equitable access to diagnostics, treatments and vaccines for every eligible country around the world (14). The COVAX facility under the vaccine pillar of the ACT Accelerator, aims to ensure an accelerated diverse portfolio of COVID19 vaccine development and manufacturing (15). The COVAX facility with Advance Market Commitment (AMC) works to incentivize the vaccine manufacturers to produce sufficient quantities of eventual COVID- 19 vaccines to ensure access by developing countries(16). As of end of November 2020, 186 countries have formally committed to join the COVAX facility (17), and Gavi reports that 92 low- and middleincome countries meet eligibility requirements for access to COVID-19 vaccines through COVAX AMC.

The COVAX facility goal is to securea sufficient number of doses of COVID-19 vaccines to cover at least $20 \%$ of a participating countries' populations and aims to deliver 2 billion doses of approved COVID-19 vaccine by the end of 2021, thus ending the acute phase of the pandemic. The facility will allocate vaccines across countries according to a value framework and will be rolled out in two phases. In the first phase, vaccines will be allocated according to population size to all participating countries at the same time, to protect those people at greatest risk of infection and of severe disease $(18,19)$. The facility is an example of global solidarity to fight together against the global pandemic.

\section{The Role of the Private Sector}

The private sector engagement and partnership has a critical role to play in the manufacturing, deployment and implementation of COVID-19 vaccination program(13).

The private sector is diverse, comprised of private hospitals, polyclinics, and smaller facilities as well as pharmacies, private insurance companies, private professional associations, private medical training institutions. As such in many countries it is a large provider of healthcare, including immunization services. To ensure adequate COVID-19 vaccination coverage and quality of services, engaging the private sector must be considered and an optimal model of publicprivate engagement should be planned in each country. This plan should include participation of private sector representative in National Immunization Technical Advisory Groups (NITAGs), with clear reporting mechanisms to ensure flow of data and its quality for monitoring and evaluation of programs; supporting immunization services and surveillance activities; engaging in training and capacity building activities, and formal agreements or contracts on COVID-19 vaccine purchase and distribution facilitating accountability and transparency.

Non-Governmental Organizations (NGOs) and other no-for-profit organizations also deliver healthcare services and immunizations in many countries and to be engaged in COVID-19 vaccine efforts in similar manner as with the private sector.

\section{FRAMEWORK FOR DECISION-MAKING}

\section{Role of National Immunization Technical Advisory Group (NITAG)}

The National Immunization Technical Advisory Groups (NITAGs) are multidisciplinary groups of national experts responsible for providing independent guidance and evidence-based advice to national policy- and decision-makers (20). As such they play a central role in Covid19 pandemic response.

NITAG members need to stay abreast of the evolving epidemiological situation of the pandemic and of COVID-19 vaccine development, as they must provide timely recommendations to policymakers on COVID19 vaccination programs, including prioritization of target groups.

The committee is expected ensure the use of a transparent procedure for decision-making regarding recommendations for future COVID19 vaccine, aiming at maintaining public trust which will be fundamental to the success of the programs before, during, and long after COVID19 vaccine implementation. NITAG recommendations also allow early engagement of different stakeholders involved in vaccine delivery to support distribution and ensure consistent communication, bringing in the private sector and NGOs. 


\section{Target Groups for Prioritization}

Studies have shown that certain age groups, mostly older adults and elderly, and people with underlying conditions and co morbidities are at a higher risk of severe COVID-19 or death, compared to children $(21,22)$. Even though some children and adolescents have been sick with COVID-19, most symptomatic and severe cases are attributed to adults. It is therefore necessary to ensure reaching high-risk groups first, once the vaccine is available when it is of limited supply and when the virus transmission is high.

There is no doubt that healthcare workers are at high to very high risk of acquiring and transmitting infection and should also be equally prioritized for COVID-19 vaccination since they play a key role in the health and well-being of the community. Protecting their health help preserve others' health as well.

WHO Strategic Advisory Group of Experts on Immunization (SAGE) on COVID-19 has recently published the values framework for the allocation and prioritization of COVID-19 vaccination(23). This document provides guidance globally on the allocation of COVID19 vaccines between countries, and also offers guidance to national authorities on allocations and prioritizing groups for vaccination within countries when the vaccine supply is scanty. The framework six principles are protection and promotion of human well-being, equal respect of all human beings, global and national equity in vaccine access and benefits, reciprocity obligation for individuals and groups within countries that bear significant additional risks and burdens of COVID-19 response for the benefit of society, and legitimacy and transparency of global and national vaccine allocation decisions.

The WHO roadmap for prioritization of COVID19 vaccine for specific priority group requires "the integration of these values with evidence and information about: 1) the status of the pandemic in the proposed implementation area; 2) the overall public health strategy for each epidemiologic setting; 3) the amount and pace of vaccine supply; 4) specific characteristics of available vaccine(s); and 5) the benefit-risk assessment for the different population groups; as well as other standard criteria used in developing SAGE recommendations (e.g., feasibility, resource use, values and preferences).
Based on these guidelines, countries can formulate strategies on how to prioritize the COVID-19 vaccine among the most vulnerable groups, and gradually transitioning to the rest of the population, until herd immunity is achieved, and virus transmission stops. It is important to consider the current COVID-19 epidemiologic trends, and the population with the highest morbidity and mortality rates, and the socioeconomic impact of the pandemic in a particular setting while developing the prioritization strategy. Based on the prioritized target population(s), potential immunization sites can be identified to ensure smooth delivery of the COVID-19 vaccine, without jeopardizing routine immunization services.

\section{Assessment of COVIID-19 Vaccine Knowledge, Attitude and Perception among Healthcare Workers and the General Public}

Having an indication on the acceptance rate of a potential COVID-19 vaccine will assist countries with planning their deployment and implementation strategies. Countries can consider conducting studies to assess the knowledge, acceptability and perception of healthcare workers and the general public towards COVID-19 vaccines. As healthcare workers are key in communicating with the general public, studies can also assess their behavior in recommending the vaccine to their patients.

The results of these studies can help the governments to address any potential barriers, and draft recommendations for successful introduction of COVID-19 vaccine once available and ensure high uptake and boost vaccine confidence.

The uptake of a future Covid19 vaccine may pose challenges as recent studies and polls showed varied positive reception with demographic and geographical disparities. For example, it was estimated $67 \%$ in the US (24), $43 \%$ in Greece (25) and $29.0 \%$ in North-Central Nigeria (26) of interviews would accept COVID-19 vaccines when available. In June 2020, a survey in 19 countries (27) showed that $71.5 \%$ of participants reported are likely to receive COVID-19 vaccine, and $61.4 \%$ reported that they would accept their employer's recommendation to do so. Differences in acceptance rates ranged from almost 90\% (in China) to less than 55\% (in Russia).

Even before the pandemic the issue of vaccine hesitancy was listed by the WHO in 2019 as one of 10 major global health threats (28). 
Therefore, this issue will need special attention and concerted effort, focused advocacy materials, training and media tools, to ensure sufficient vaccination coverage to stop the pandemic is achieved.

\section{PROGRAMMATIC AND OPERATIONAL ISSUES Supply Chain of COVID-19 Vaccine}

A well-functional cold chain with sufficient capacity will be required for efficient supply and delivery of COVID-19 vaccines (29). Current available vaccines differ in their temperature requirements. Pfizer and BioNTech vaccine requires stringent temperature (up to $-80^{\circ} \mathrm{C}$ ), which poses challenges to existing supply chain in some parts of the world. Moderna AstraZeneca's COVID-19 vaccines, can be stored and transported at normal refrigerated temps of 2 degrees to $8^{\circ} \mathrm{C}$. Other vaccines in the pipelines may have different requirements.

No matter which vaccine(s) is licensed for use, countries are advised to assess and intensify, if necessary, their supply chain capacity to ensure its safety and stability and strengthen effective vaccine management, including resilient logistical infrastructures.

Training of Immunization Managers and Different Cadres of Healthcare Workers

Immunisation managers, both at the national and regional levels, are key for the successful planning and implementation of the COVID-19 vaccination program. The strong managerial and planning skills, including monitoring of the immunization system, and ensuring disease surveillance work effectively. Healthcare workers, including vaccinators and community health workers, require robust skills in correct handling and administration of the new vaccine, cold chain, vaccines and safe-injection equipment management, management of adverse events and data recording and reporting and also on how to organize safe immunization sessions during COVID-19 outbreaks. Good interpersonal communication skills are also required because they are in direct contact with the community and are key in building public trust for vaccination. Therefore, it is important totrain and update both immunization managers and healthcare workers on different aspects of COVID-19 vaccination programs and provide this team with necessary tool to do their duties effectively and safe

\section{Community Engagement}

Community engagement will create awareness about COVID-19 vaccine and support demand creation. It should be use do clearly communicate risks and the benefits to the population, and address any concerns prior to the implementation of the vaccination program $(40,41)$. Community and religious leaders, NGOs and community associations, can be mobilized to build trust in the vaccination programs, so that people will be confident to get vaccinated, tackle misinformation and disinformation and to ensure their involvement and participation. Understanding the reasons, if any, why people may not in favor of vaccination or lack of motivation or desire for immunization, will be important to be able to tackle them, while maximizing vaccine uptake. A survey or a situation analysis, prior to the launch of COVID-19 vaccination, among different segments of the community on their knowledge, attitudes and practices towards COVID-19 vaccine, could identify these issues and define appropriate solutions, including community and advocacy and communications strategies and channels(30).

\section{Post-Marketing Surveillance}

Post-marketing surveillance is crucial to ensure that the vaccines that are being used are safe, do not induce long-term adverse reactions, nor induce unusual or rare adverse reactions in a small sub-group of the population or unintended side effects. Since COVID-19 vaccines are being developed in an unprecedented short time frame of 12-18 months, it is not possible to study the long-term adverse reaction (if any) associated with the administration of the vaccine. Therefore, post-marketing surveillance and 'real time' monitoring activities is important for thorough safely and efficacy monitoring to identify and document any adverse event that may arises, to maintain public trust by effective communication about these event and their possible link with vaccination or not, and to implement constructive interventions to the COVID-19 vaccination program $(31,32)$.

Structured post marketing surveillance include, in addition to safety monitoring, monitoring activities of vaccine effectiveness, coverage, and impact.

\section{CONCLUSION}

As COVID-19 vaccines start to be approved and licensed for use, it is paramount for the countries to prepare for the effective implementation of COVID-19 vaccination program and harness maximum benefits once the COVID-19 vaccine is available. Failure to 
address the key challenges and considerations for COVID-19 vaccine implementation outlined in this paper, including regulatory and legal issues, frameworks for decision-making and programmatic and operational matters, can result in ineffective vaccination programs, thus prolonging the current global health crisis and its heavy toll on human, social and economic interactions.

The ongoing pandemic has made us truly realize the importance and necessity of investment in vaccine development and manufacturing capacities at the regional and country level, besides, having a reliable and well prepared logistic and supply chain system ready to deploy the manufactured vaccines once authorized. Local vaccine production provides significant benefits for countries not only during the pandemic but also in the manufacturing capacity of other vaccines as well (33). This can ultimately cut down the vaccine prices and can be one step forward towards achieving Universal Health Coverage (UHC). It also highlighted the vital matter of fair and equitable access to global public goods such as pandemic vaccine. The COVAX facility is an example of global solidarity to fight together against the global pandemic and should be supported and financed to achieve its goal of ensuring access and fair allocation of COVID-19 vaccines to developing countries.

\section{REFERENCES}

[1] United Nations Development Program (UNDP). 2020. Socio-economic impact of COVID-19 [Internet]. UNDP. [Accessed on 25 Aug 2020]. Available from: https://www.undp. org/content/undp/en/home/ coronavirus/socioeconomic-impact-of-covid-19.html

[2] World Health Organization (WHO). 2020. WHO Coronavirus Disease (COVID-19) Dashboard. Available from: https://covid19. who.int/

[3] World Health Organization (WHO). Draft landscape of COVID-19 candidate vaccines. Updated on Sept 30 2020. [Date Accessed 2020 Sept 30] Available from: https://www.who.int/ publications $/ \mathrm{m} /$ item/draft-landscape-of-covid19-candidate-vaccines2.

[4] Pfizer. 2020. Our COVID-19 Vaccine Study What's Next? Accessed on 21 November 2020. Available at https://www.pfizer.com/news/hottopics/our_covid_19_vaccine_study_what_s_next

[5] Moderna.2020. Moderna's COVID-19 Vaccine Candidate Meets its Primary Efficacy Endpoint in the First Interim Analysis of the Phase 3
COVE Study. Accessed on November 16, 2020. Available from https://investors. modernatx. com/news-releases/news-releasedetails/modernas-covid-19-vaccine-candidatemeets-its-primary-efficacy.

[6] Voysey M, Clemens SAC, Madhi SA, Weckx LY, Folegatti PM,AleyPKet al. Safety and efficacy of the ChAdOx1 nCoV-19 vaccine (AZD1222) against SARS-CoV-2: an interim analysis of four randomised controlled trials in Brazil, South Africa, and the UK. https:// doi.org/10.1016/S0140-6736(20)32661-1

[7] Randolph HE, Barreiro LB. Herd Immunity: Understanding COVID-19. Immunity. May 2020;52(5):737-41. https://doi.org/10.1016/ j.immuni.2020.04.012

[8] European Commission (EC). Communication from The Commission. Preparedness for COVID-19 vaccination strategies and vaccine deployment. Brussels. Accessed on 14 December 2020. Available from https://eurlex.europa.eu/legalcontent/EN/TXT/?uri=COM:2020:0680:FIN

[9] World Health Organization (WHO). Concept for fair access and equitable allocation of COVID-19 health products. Final working version 9 September 2020. Accessed on 14 December 2020. Available from https:// www.who.int/publications/m/item/fairallocation-mechanism-for-covid-19-vaccinesthrough-the-covax-facility

[10] World Health Organization (WHO).Interim Guidance on developing a national deployment and vaccination plan for COVID-19 vaccines. Available from https://www.who.int/publica tions/i/item/WHO-2019-nCoV-Vaccine_ deployment-2020.1

[11] U.S. Food and Drug Administration, Center for Biologics Evaluation and Research. Development and Licensure of Vaccines to Prevent COVID-19: Guidance for Industry. Jun 2020 [Accessed on 2020 Oct 1]. Available from:https://www.fda.gov/regulatory-

information/search-fda-guidance-documents/ development-and-licensure-vaccines-preventcovid-19

[12] Magenta M. What lessons the unequal distribution of the H1N1 vaccine leaves for the coronavirus 10 years ago. Web24 News. 2020 Aug 13 [Accessed on 2020 Oct 1]. Available from: https://www.web24. news/u/ 2020/ 08/ what-lessons-the-unequal-distribution-of-theh1n1-vaccine-leaves-for-the-coronavirus-10years-ago.html

[13] Callaway E. The unequal scramble for coronavirus vaccines- by the numbers. Nature. 
2020; 584(7822):506-7. Available from: https://www.nature.com/articles/d41586-02002450-x

[14] World Health Organization (WHO). The Access to COVID-19 Tools (ACT) Accelerator. 2020.[ Accessed on1 Oct 2020]. Available from: https://www.who.int/initiatives/actaccelerator

[15] The Global Vaccine Alliance (GAVI). COVAX Facility. [Accessed on 30 Sept 2020]. Available from: https://www.gavi.org/covax-facility

[16] The Global Vaccine Alliance (GAVI). The GAVI COVAX AMC An Investment opportunity. 2020 [Accessed on 1 Oct 2020]. Available from: https://www.gavi.org/ news/ media-room/gavi-launches-innovativefinancing-mechanism-access-covid-19-vaccines

[17] The Global Vaccine Alliance (GAVI). New collaboration makes further 100 million doses of COVID-19 vaccine available to low- and middle-income countries. 2020 [Accessed on 1 Oct 2020]. Available from: https://www.gavi. org/news/media-room/new-collaborationmakes-further-100-million-doses-covid-19vaccine-available-low

[18] U.S. Food and Drug Administration, Center for Biologics Evaluation and Research. Development and Licensure of Vaccines to Prevent COVID-19: Guidance for Industry. Jun 2020 [Accessed on 1 Oct 2020]. Available from:https://www.fda.gov/regulatory-information/ search-fda-guidance-documents/developmentand-licensure-vaccines-prevent-covid-19

[19] International Coalition of Medicines regulatory Authorities (ICMRA). ICMRA COVID-19 Treatments and Clinical Trials Workshop \#2 Summary. 2020 [Accessed on 1 Oct 2020]. Available from: http://www.icmra. info/ drupal/ news/20july2020/summary

[20] World Health Organization (WHO). National advisory committees on immunization [Internet]. WHO. World Health Organization; 2019 [Accessed on 1 October 2020]. Available from: http://www.who.int/ immunization/ sage/ national_advisory_committees/en/

[21] 21.Patel KP, Vunnam SR, Patel PA, Krill KL, Korbitz PM, Gallagher JP, et al. Transmission of SARS-CoV-2: an update of current literature. Eur J Clin Microbiol Infect Dis. Jul 2020;1-7. DOI: https://doi.org/10.1007/s10096 -020-03961-1

[22] Peterson E, Koopmans M, Go U, Hamer DH, Petrosillo N, Castelli F, et al. Comparing SARS-CoV-2 with SARS-CoV and influenza pandemics. Lancet Infect Dis. Sep 2020; 20(9):e238-44 https://doi.org/ 10.1016/S14733099(20)30484-9

[23] World Health Organization (WHO). WHO SAGE values framework for the allocation and prioritization of COVID-19 vaccination. 2020 [Accessed on 1 Oct 2020]. Available from: https://apps.who.int/iris /bitstream/ handle/ 106 65/334299/WHO-2019-nCoV-SAGE_

Framework-Allocation_and_prioritization-20 20.1-eng.pdf

[24] MalikAA, McFaddenSM, ElharakeJ, Omer S.B. Determinants of COVID-19 vaccine acceptance in the US. Research Paper| Volume 26, 100495, September 01, 2020. DOI: https:// doi.org/10.1016/ j.eclinm.2020.100495

[25] Papagiannis D, Malli F, Raptis DG, PapathanasiouIV, FradelosEC, Daniil Z, Rachiotis G and Gourgoulianis KI. Assessment of Knowledge, Attitudes, and Practices towards New Coronavirus (SARS-CoV-2) of Health Care Professionals in Greece before the Outbreak Period. International Journal of Environmental Research and Public Health. Int. J. Environ. Res. Public Health 2020, 17, 4925; doi:10.3390/ijerph17144925

[26] Reuben RC, Danladi MMA, Saleh DA, Ejembi PE. Knowledge, Attitudes and Practices Towards COVID-19: An Epidemiological Survey in North-Central Nigeria [published online ahead of print, $2020 \mathrm{Jul}$ 7]. J Community Health. 2020;1-14. doi:10.1007/s10900-02000881-1

[27] Lazarus, J.V., Ratzan, S.C., Palayew, A. et al. A global survey of potential acceptance of a COVID-19 vaccine. Nat Med (2020). https://doi.org/10.1038/s41591-020-1124-9

[28] World Health Organization (WHO). 2019. Ten threats to global health in 2019. Accessed on 1 Oct 2020. Available fromhttps://www. who.int/ news-room/spotlight/ten-threats-to-globalhealth-in-2019

[29] World Health Organization (WHO). Vaccine management and logistics support. Updated on Feb 2020 [Accessed on 1 Oct 2020]. Available from: http://www.who.int/ immunization/ programmes_systems/supply_ chain/ resources/ tools/en/

[30] French J, Deshpande S, Evans W, Obregon R. Key Guidelines in Developing a Pre-Emptive COVID-19 Vaccination Uptake Promotion Strategy. Int J Environ Res Public Health. 2020;17(16):5893. https://doi.org/10. 3390/ ije rph17165893

[31] World Health Organization (WHO). Post marketing surveillance. [Accessed on 1 Oct 
Country Level Preparation for the Deployment of COVID-19 Vaccine

2020]. Available from: http://www.who.int/ medicines/regulation/ssffc/pms/en/

[32] World Health Organization (WHO). AEFI detection. [Accessed on 1 Oct 2020]. Available from: http://www.who.int/ vaccine_safety/ initiative/detection/en/
[33] Jadhav S, Gautam M, Gairola S. Role of vaccine manufacturers in developing countries towards global healthcare by providing quality vaccines at affordable prices. Clin Microbiol Infect. 2014;20:37-44. https://doi.org/10.11 11/ $1469-0691.12568$

Citation: Salah T. Al Awaidy \& Chadia Wannous, "Country Level Preparation for the Deployment of COVID-19 Vaccine”, International Journal of Research Studies in Medical and Health Sciences. 2021; 6(2): 1-8. DOI: https://doi.org/10.22259/ijrsmhs.0602001

Copyright: () 2021 Salah T. Al Awaidy \& Chadia Wannous, This is an open-access article distributed under the terms of the Creative Commons Attribution License, which permits unrestricted use, distribution, and reproduction in any medium, provided the original author and source are credited. 